\title{
IMPLEMENTAÇÃO DE UM SISTEMA DE MONITORAMENTO DAS CONDIÇÕES AMBIENTAIS BASEADO EM ARDUINO
}

Eric Freitas de Abreu

Graduando em Astronomia. Estagiário no Inmetro. eabreu@inmetro.gov.br

Rodrigo Maia Tavares Mestre em Engenharia. Pesquisador-Tecnologista no Inmetro. rmtavares@inmetro.gov.br

Renato Silveira da Costa

Graduando em Licenciatura em Física, Técnico em Telecomunicações. Técnico no Inmetro rscosta@inmetro.gov.br

Vinicius Coutinho de Oliveira Mestre em Engenharia. Pesquisador-Tecnologista no Inmetro. vcoutinho@inmetro.gov.br

Helio Ricardo Carvalho Doutor em Física. Pesquisador-Tecnologista no Inmetro. hrcarvalho@inmetro.gov.br

Regis Pinheiro Landim Doutor em Engenharia Elétrica. Pesquisador-Tecnologista no Inmetro rplandim@inmetro.gov.br

\section{RESUMO}

Este artigo trata da construção, programação e implementação de um Sistema de Monitoramento das Condições Ambientais (SMCA) no Laboratório de Metrologia Elétrica Quântica do Instituto Nacional de Metrologia, Qualidade e Tecnologia (Inmetro). O SMCA tem o objetivo de monitorar temperatura e umidade, permitindo apurar se as mesmas estão dentro dos limites adequados para se realizar a calibração de padrões de referência de tensão contínua baseados em diodos Zener. Adicionalmente, o SMCA também está sendo usado para monitorar a pressão atmosférica, o que será usado em estudos sobre a influência da pressão atmosférica em sistemas de padronização de tensão contínua. O SMCA foi programado nas linguagens $C / C++$ e VBA, proporcionando interação entre microcontrolador (Arduino) e Excel. Desde julho de 2018, o SMCA vem medindo e registrando suas medições que, em análise prévia, se encontram em níveis aceitáveis em comparação a padrões rastreados ao Sistema Internacional de Unidades (S.I.).

Palavras-chave: Arduino. Calibração. Condições Ambientais. Metrologia. Zeners.

\begin{abstract}
This article deals with the construction, programming and implementation of a Monitoring System for Environmental Conditions (SMCA) in the Quantum Electrical Metrology Laboratory of the National Institute of Metrology, Quality and Technology (Inmetro). The SMCA aims at monitoring temperature and humidity, determining if they are within the proper limits to perform calibrations
\end{abstract}


of reference standards of continuous voltage based on Zener diodes. Additionally, the SMCA also is being used to monitor atmospheric pressure, which will allow us to use it to support studies on the influence of atmospheric pressure on primary systems of continuous voltage. The SMCA was programmed on the languages $\mathrm{C} / \mathrm{C}++$ and $\mathrm{VBA}$, providing interaction between the microcontroller (Arduino) and Excel. Since July/2018, the SMCA has been measuring and recording its measurements, which, in previous analyses, are within acceptable levels compared to standards tracked to the International System of Units (S.I.).

Keywords: Arduino. Calibration. Environmental Conditions. Metrology. Zeners.

\section{INTRODUÇÃO}

Este artigo trata da construção, programação e implementação de um Sistema de Monitoramento das Condições Ambientais (SMCA) baseado em Arduino. Esse sistema tem sua necessidade evidenciada devido aos processos de calibração que ocorrem no Laboratório de Metrologia Elétrica Quântica (Lameq) do Inmetro. O Lameq fica no topo da cadeia de rastreabilidade da unidade de tensão elétrica (volt) no Brasil, e para disseminar esta unidade utilizam-se dois sistemas: (a) o de padronização primária de tensão contínua, baseado no Efeito Josephson (um efeito de supercondutividade, no qual ocorrem degraus de tensão elétrica com exatidão da ordem de $10^{-9} \mathrm{~V}$ ); e (b) o secundário, composto por padrões de tensão contínua (que produzem tensões de referência em $10 \mathrm{~V}$ e 1,018 V, baseados no Efeito Zener), também chamados de "padrões Zener". Para que calibrações com estes sistemas tenham validade, é necessário que as condições ambientais estejam dentro de certos níveis, estipulados previamente por normas do Inmetro: (a) $(23,0 \pm 2,0)^{\circ} \mathrm{C}$ para a temperatura; e (b) $(57,5 \pm 12,5)$ \%ur para umidade relativa (LANDIM, 2018, p. 6).

Inicialmente, apenas temperatura e umidade eram medidas e monitoradas, deixando de lado a pressão atmosférica, já que na norma mencionada acima não há definição de limites para os quais a pressão atmosférica seja considerada prejudicial às calibrações. Posteriormente, foi incluído no SMCA o monitoramento da pressão atmosférica, para possibilitar estudos de influência desta grandeza nas calibrações executadas no Lameq. O SMCA tem, portanto, o objetivo de ser uma solução integrada, medindo e monitorando todas as três grandezas em um único sistema e também de apoiar estudos acerca da influência da pressão atmosférica nas calibrações realizadas no Lameq.

O Arduino é uma plataforma de prototipagem eletrônica de hardware livre e de placa única (VINCENZI, 2015), que vem ganhando espaço pelo baixo custo em relação a outras plataformas mais complexas, pela facilidade de programação e pela acessibilidade de componentes. Além 
disso, no caso deste trabalho, o principal motivo pela escolha do SMCA ser baseado em Arduino foi a possibilidade de integrá-lo ao Microsoft Excel, já que as medições feitas pelo SMCA precisavam ser registradas em uma planilha do Excel, solução utilizada por todos os demais sistemas do laboratório.

\section{METODOLOGIA}

O SMCA funciona com base em dois macroblocos de programação: (a) o bloco de programação do Arduino, que faz os sensores medirem e enviarem os dados tanto ao computador ao qual o Arduino está conectado via porta USB (Universal Serial Bus), quanto ao display onde aparecem as medições, utilizando a linguagem $\mathrm{C} / \mathrm{C}++$, e; (b) o bloco de programação do Excel, que recebe as medições e as registra em uma planilha, por meio da linguagem VBA (Visual Basic for Applications).

\subsection{Hardware do SMCA}

O SMCA emprega dois sensores: um de temperatura e umidade relativa, modelo DHT22 (LIU, 2010), e outro de temperatura e pressão atmosférica, modelo BMP280 (BOSCH SENSORTEC, 2015). Entretanto, o BMP280 foi utilizado somente para medir pressão atmosférica, já que a precisão da medição de temperatura do DHT22 é melhor em relação ao BMP280.

O diagrama elétrico do SMCA, elaborado com o programa de código aberto Fritzing (FRITZING, 2016), é mostrado na Figura 1. A alimentação de $+5 \vee$ do Arduino e periféricos é provida pela interface USB. A comunicação com os periféricos é realizada pelas saídas digitais do Arduino. Além disso, são utilizados alguns componentes discretos: um LED verde (LED1) associado a um resistor limitador de corrente de $1 \mathrm{k} \Omega$ (R1), que indica se o SMCA está energizado; um LED vermelho (LED2) associado a outro resistor limitador de corrente de $1 \mathrm{k} \Omega$ (R2), que acende se uma das variáveis ambientais estiver fora dos limites especificados, e; uma malha divisora de tensão composta pelos resistores R3 e R4, que define uma tensão de aproximadamente $680 \mathrm{mV}$ no pino Vo do display, determinando o contraste luminoso desejado. A lista de materiais completa é apresentada na Tabela 1. Cabe salientar que, por se tratar ainda de um protótipo, o SMCA foi montado em protoboard. Na Figura 2, pode-se ver uma foto do protótipo construído. 
Figura 1 - Diagrama elétrico do SMCA

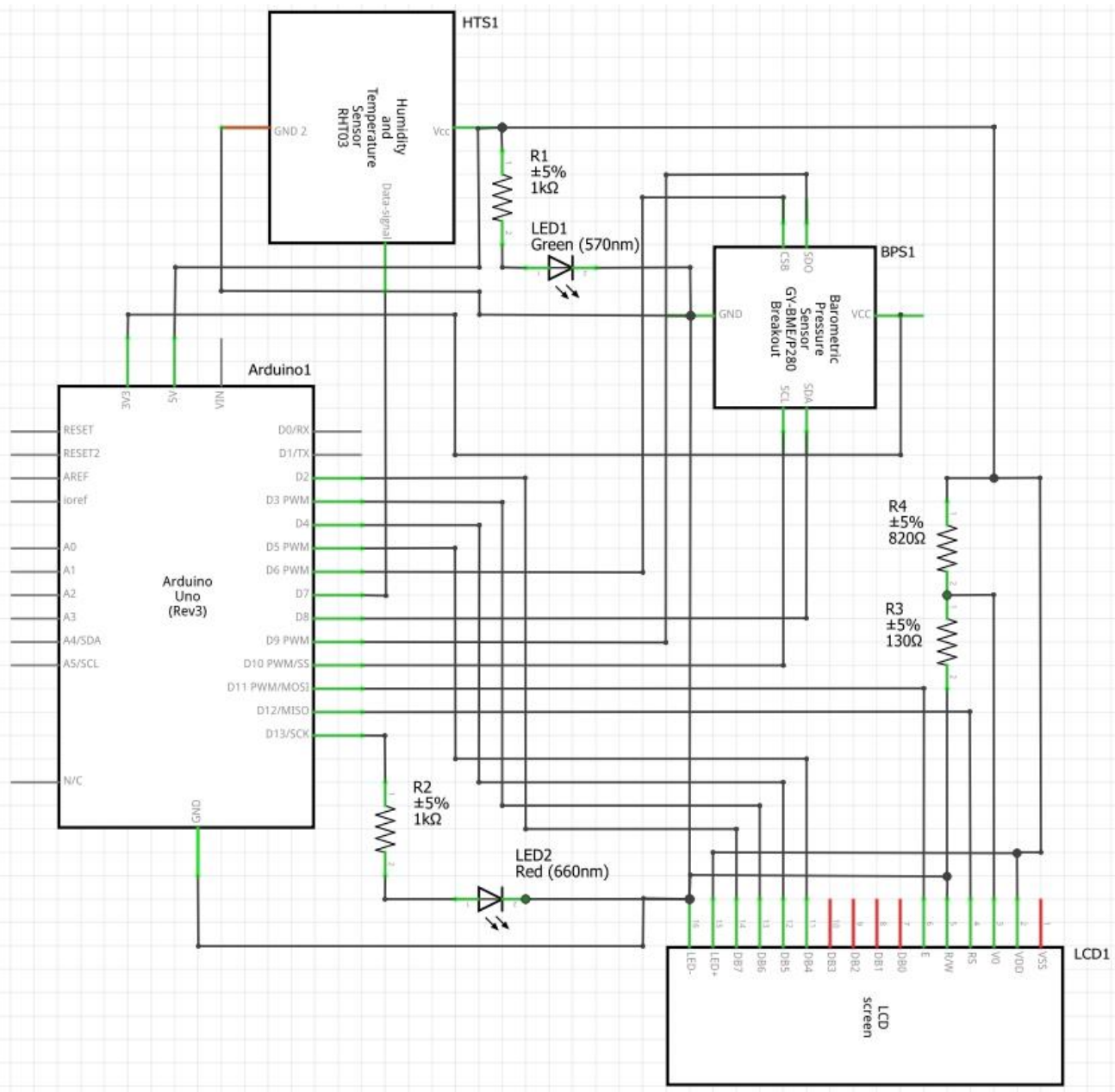

Figura 2 - Protótipo do SMCA

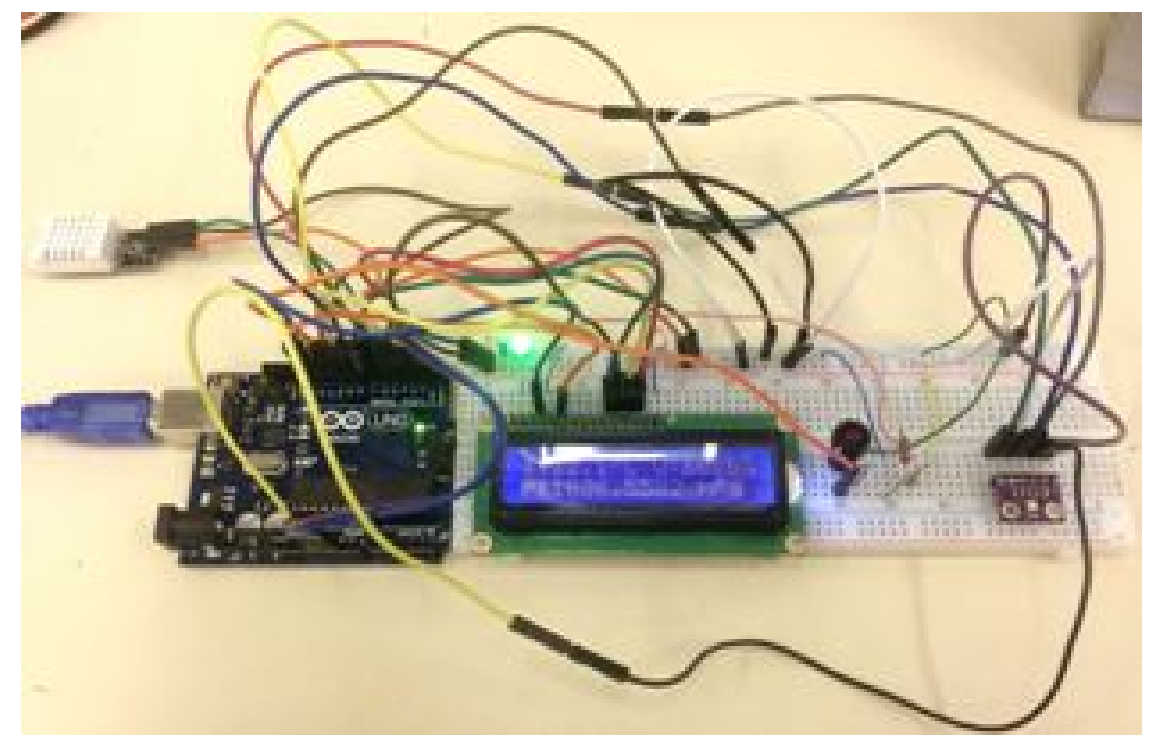


Tabela 1 - Lista de materiais do SMCA.

\begin{tabular}{c|c|c}
\hline Componente & Marca e modelo & Custo \\
\hline Arduino1 & Arduino Uno (Rev3) & $\mathrm{R} \$ 52,00$ \\
\hline BPS1 & Sensor barométrico de pressão BMP280 & $\mathrm{R} \$ 21,00$ \\
\hline HTS1 & Sensor de temperatura e umidade DHT22 & $\mathrm{R} \$ 35,00$ \\
\hline LCD1 & Display de LCD & $\mathrm{R} \$ 15,00$ \\
\hline LED1 & LED verde & $\mathrm{R} \$ 0,50$ \\
\hline LED2 & LED vermelho & $\mathrm{R} \$ 0,50$ \\
\hline R1, R2 & Resistores de $1 \mathrm{k} \Omega \times 1 / 4 \mathrm{~W}$ & $\mathrm{R} \$ 0,40$ \\
\hline R3 & Resistor de $130 \Omega \times 1 / 4 \mathrm{~W}$ & $\mathrm{R} \$ 0,20$ \\
\hline R4 & Resistor de $820 \Omega \times 1 / 4 \mathrm{~W}$ & $\mathrm{R} \$ 0,20$ \\
\hline- & Jumpers e cabos & $\mathrm{R} \$ 10,00$ \\
\hline- & Protoboard $(400$ pontos) & $\mathrm{R} \$ 12,00$ \\
\hline & & $\mathrm{R} \$ 146,80$ \\
\hline
\end{tabular}

\subsection{Software do SMCA - Bloco de programação do Arduino}

O programa em Arduino cuida das medições feitas pelos sensores anteriormente mencionados. As medições são enviadas a cada $3 \mathrm{~s}$ para o display (Figura 3), após passarem por etapas de "segurança" do código, as quais têm o propósito de impedir o envio de medições inválidas (por falha dos sensores, por exemplo) para o display, e, consequentemente, para o Excel. O display serve como mostrador e, também, como aviso para quem estiver usando essas medições, já que quando as grandezas ultrapassam os limites especificados, um LED vermelho se acende e a informação "Imp." (que equivale à "Imprópria", isto é, as condições estão "Impróprias para calibração") é exibida no display, no campo "valor" da grandeza a qual extrapolou o limite (Figura 4). O display alterna, num intervalo de $1 \mathrm{~s}$, entre o valor da grandeza e a mensagem de "Imp.".

Figura 3 - Grandezas enviadas para o display

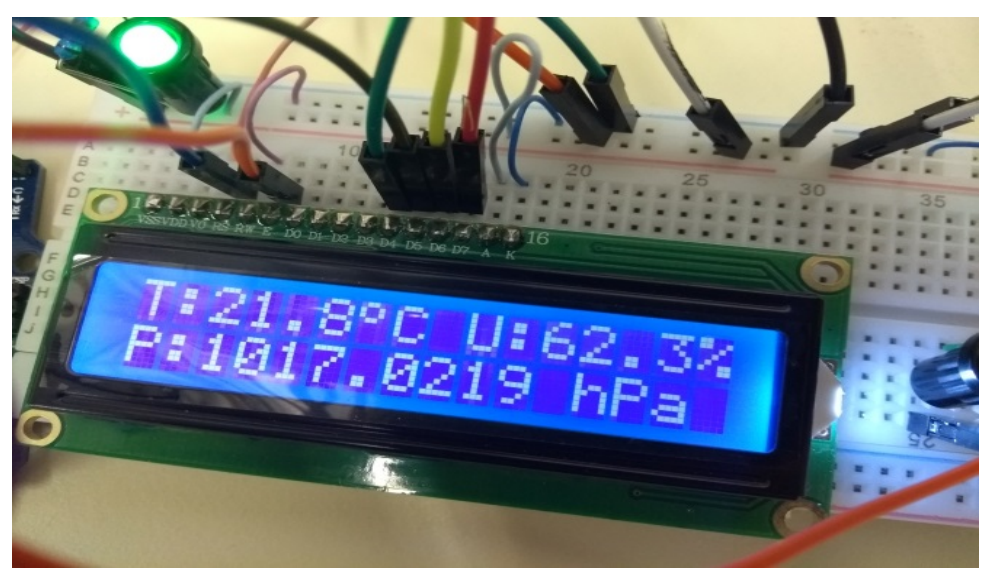


Figura 4: Exemplo de situação fora dos limites especificados, na qual a umidade está "Imprópria para Calibração"

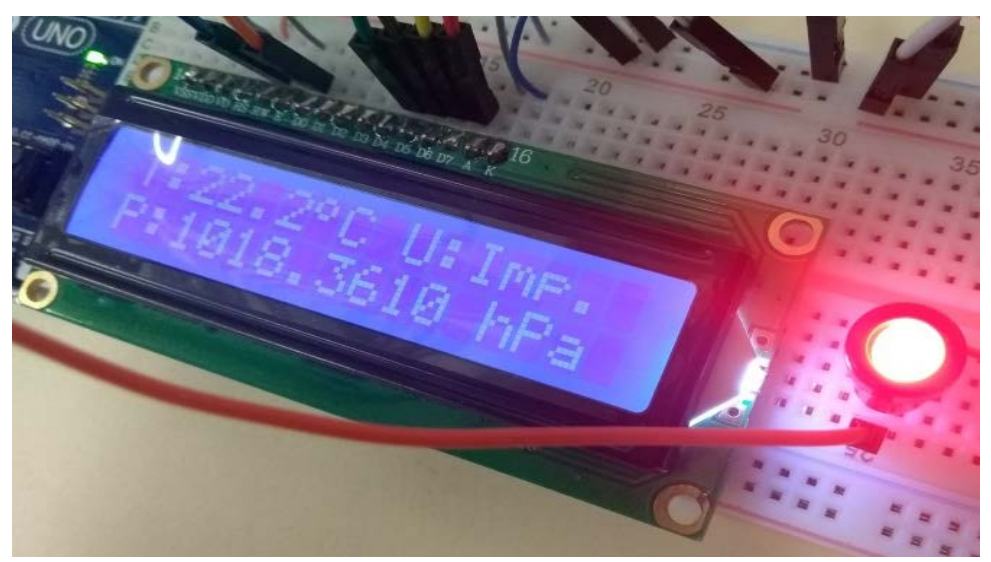

\subsection{Software do SMCA - Bloco de programação do Excel}

Embora sejam enviadas pelos sensores a cada $3 \mathrm{~s}$, as medições são registradas no Excel num intervalo de cerca de $60 \mathrm{~s}$ e, além de seguirem os processos de "segurança" do Arduino, passam por outro processo similar para que os dados não sejam registrados em planilha erroneamente.

A planilha automatizada possui dois botões, "Executar" e "Parar", associados a macros em VBA. Para iniciar o registro das medições, clica-se em "Executar"; a primeira medição é registrada automaticamente, enquanto as demais possuem um delay de $60 \mathrm{~s}$. As medições continuam sendo registradas até que o botão "Parar" seja acionado ou até o final do dia ( 23 h 59 min 59 s). Após a interrupção é gerada uma tabela cujo nome é a data da medição (ex.: “07.09.2018"), que tem as medições registradas e respectivos horários, como exemplificado na Tabela 2, e um gráfico abrangendo todas as medições da determinada grandeza (Figura 5). Vale salientar que, ao serem finalizadas as medições do dia e ser criada a tabela, as medições continuam a ser registradas, mas com a data do dia seguinte.

Tabela 2 - Exemplo do registro de medição gerado pelo programa

\begin{tabular}{c|c|c|c}
\hline Hora & Temperatura $\left({ }^{\circ} \mathbf{C}\right)$ & $\begin{array}{c}\text { Umidade Relativa } \\
\text { (\%) }\end{array}$ & $\begin{array}{c}\text { Pressão } \\
\text { Atmosférica (hPa) }\end{array}$ \\
\hline 00:00:49 & 22,80 & 63,10 & 1020,7294 \\
\hline $00: 01: 51$ & 22,80 & 63,10 & 1020,7267 \\
\hline $00: 02: 54$ & 22,80 & 63,10 & 1020,6674 \\
\hline $00: 03: 57$ & 22,80 & 63,10 & 1020,7380 \\
\hline
\end{tabular}


Figura 5 - Exemplo de gráfico gerado pelo programa

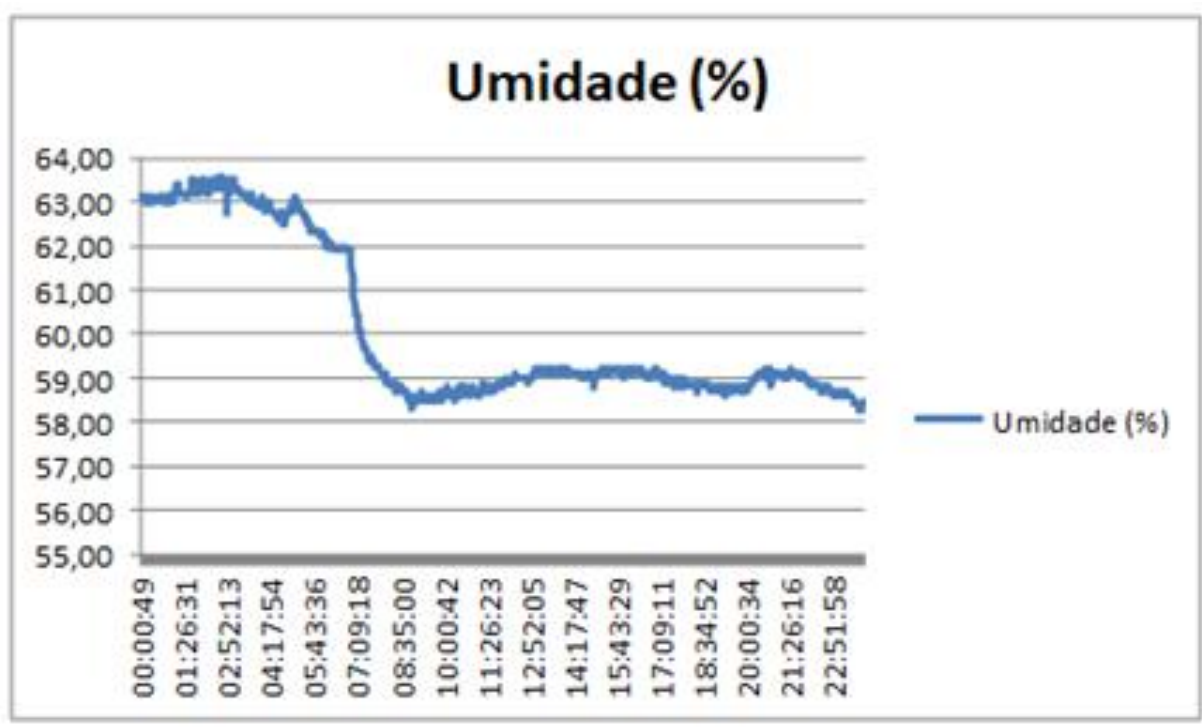

\section{RESULTADOS}

Ao comparar as medições do SMCA com o termohigrômetro e o barômetro, padrões do Lameq (rastreados ao S.I.), percebe-se que há uma pequena divergência entre as medições. Para temperatura, o erro máximo (em módulo) foi de $1,09{ }^{\circ} \mathrm{C}$ e o mínimo de $0,06{ }^{\circ} \mathrm{C}$; para umidade relativa, o erro máximo (em módulo) foi de 4,16 \%ur e o mínimo de 0,09 \%ur. Espera-se que estes erros máximos diminuam com a introdução de um fator de correção nos valores medidos, pois os resultados em temperatura ficaram o dobro do máximo especificado pelo fabricante, quais sejam, $\pm 0,5 \%$ em temperatura e $\pm 5 \%$ em umidade (LIU, 2010). Sendo assim, é possível afirmar que as medições entre o SMCA e os padrões são compatíveis. Portanto, o SMCA serve ao propósito de monitorar as condições ambientais do Lameq e, futuramente, de se estudar a influência da pressão atmosférica nos sistemas de calibração já mencionados.

\section{CONSIDERAÇÕES FINAIS}

Neste artigo, foram descritas a construção do hardware, os blocos de programação e a implementação do Sistema de Monitoramento das Condições Ambientais, ou SMCA, destinado a auxiliar no cumprimento de normas do laboratório e realizar futuras pesquisas acerca dos efeitos das condições ambientais nas calibrações do Lameq. 
O SMCA é uma solução de baixo custo de hardware (cerca de $\mathrm{R} \$ 150,00$ em peças), o que torna viável o seu uso em laboratórios e instalações que necessitam monitorar/registrar suas variáveis ambientais.

O tempo de desenvolvimento da presente versão de software foi de 6 semanas. (cerca de 130 horas-homem). Está em andamento a fase de preparação da documentação necessária à solicitação do registro do software junto ao Instituto Nacional da Propriedade Industrial (INPI).

Como continuação deste trabalho, apontam-se: (a) desenho e confecção de placa de circuito impresso e montagem em gabinete apropriado; (b) caracterização metrológica (com avaliação das incertezas de medição) e calibração oficial do sistema; e (c) ampliação das grandezas a serem monitoradas pelo SMCA: por exemplo, adição de um medidor de campo magnético com vistas à realização de estudos em outros sistemas de calibração do Lameq não mencionados neste artigo, tal como o sistema de padronização primária de resistência elétrica baseado no Efeito Hall Quântico.

\section{REFERÊNCIAS}

BOSCH SENSORTEC. BMP280 Digital Pressure Sensor Datasheet. Bosch Sensortec GmbH, Alemanha, 2015.

FRITZING. Fritzing Software Tool. Friends-of-Fritzing Foundation, Alemanha, 2016. Disponível em http://fritzing.org/download/0.9.3b/. Acesso em 06 ago. 2018.

JOSEPHSON, B.D. Discovery of tunnelling supercurrents. Estados Unidos, Proc. IEEE, 1974.

LANDIM, R.P.; DA COSTA, R.S.; TAVARES, R.M. NIT-LAMEQ-003: calibração de padrão de referência de tensão contínua. Inmetro, Duque de Caxias, 2018.

LIU, T. Digital-output relative humidity \& temperature sensor/module DHT22 Datasheet. Aosong Electronics Co.,Ltd., 2010.

VINCENZI, F. Arduino. Sistemas Embarcados (página web), 2015. Disponível em: www.omegaflix.com/arduino. Acesso em 12 set. 2018. 